\title{
Correction: Planck stars as observational probes of quantum gravity
}

Carlo Rovelli

Nature Astronomy 1, 0065 (2017); published 2 March 2017; corrected 6 March 2017.

In the version of this Comment originally published, the HTML version of equation (2) was incorrect and the hyperbolic sine term should have read: 'sinh ${ }^{-1}$. This has been corrected in all versions of the Comment. 